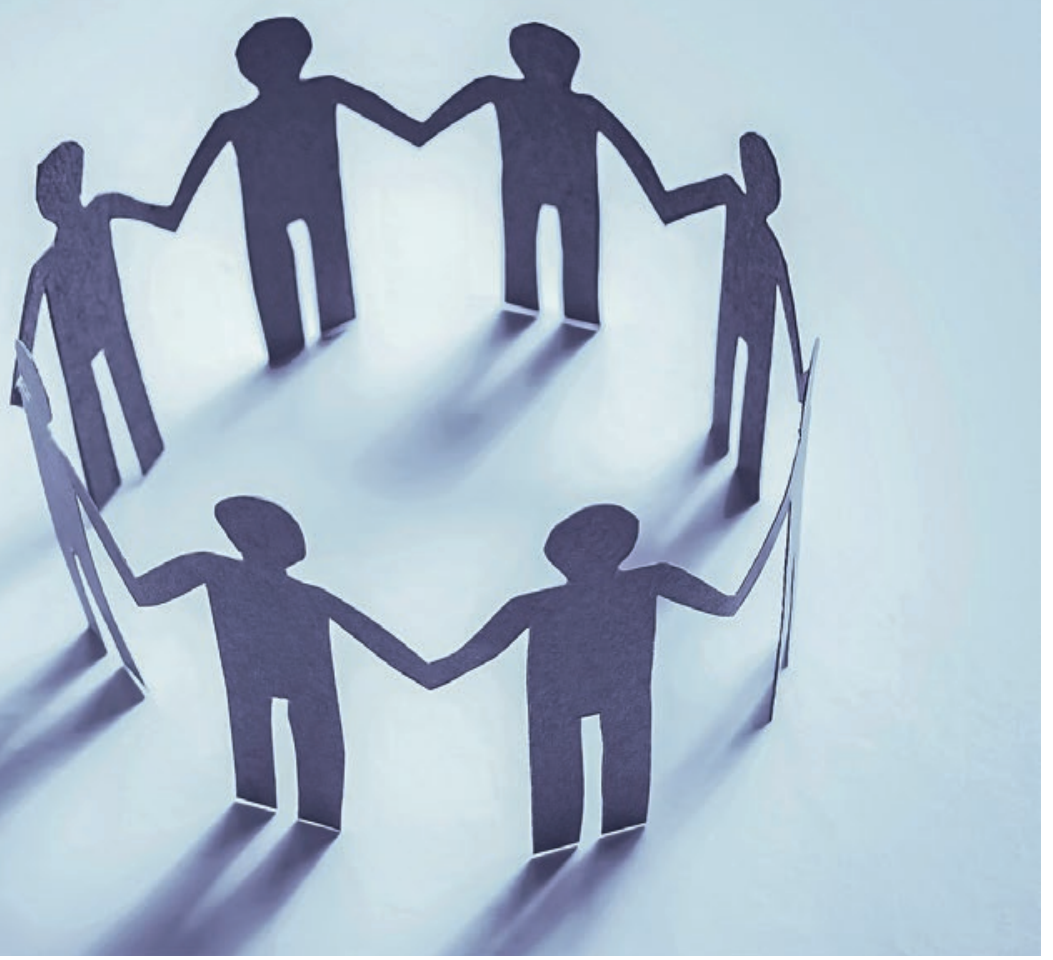

\title{
Une base solide pour aller plus loin
}

Gert Ulricha ${ }^{a}$ Sylvia Kaap-Fröhlich ${ }^{b}$

a Dr phil., M.A., fondation Careum, Zurich; ${ }^{b}$ Dr rer. nat., MBA, fondation Careum, Zurich

On n'imagine plus aujourd'hui les soins sans interprofessionnalité. Les conditions d'une collaboration axée sur les besoins entre les différents groupes professionnels sont fondamentalement bonnes en Suisse. Ce prérequis favorable doit maintenant être mis à profit pour promouvoir et ancrer l'interprofessionnalité dans le système de santé helvétique.

«Ayant eu besoin de soins pendant une longue période, j’ai remarqué que les professionnels de santé ne parlaient pas souvent entre eux [...] J'ai régulièrement vécu des situations où je devais tout reprendre depuis le début pour chaque professionnel de santé. Cela m’a rendue méfiante vis-à-vis de l'institution qu'est l'hôpital» [1]. Cette citation d'une patiente suisse montre qu'il y a encore beaucoup à faire dans le domaine des soins de santé. Mais au-delà de la satisfaction et de la sécurité des patients, le système de santé sera confronté à d'autres défis croissants dans les années à venir comme l'évolution démographique, l'augmentation des maladies chroniques, l'explosion des coûts et le manque de main-d'œuvre qualifiée dans les professions de la santé et de la médecine.

Il ne fait aucun doute que la collaboration interprofessionnelle et la formation interprofessionnelle, qui lui ouvre la voie, ne peuvent pas être des remèdes miracu-

\section{Définition de l'interprofessionnalité}

L'Organisation mondiale de la Santé (OMS) définit la collaboration (interprofessionnelle) comme suit: "Collaboration occurs when two or more individuals from different backgrounds with complementary skills interact to create a shared understanding that none had previously possessed or could have come to on their own» [3]. 


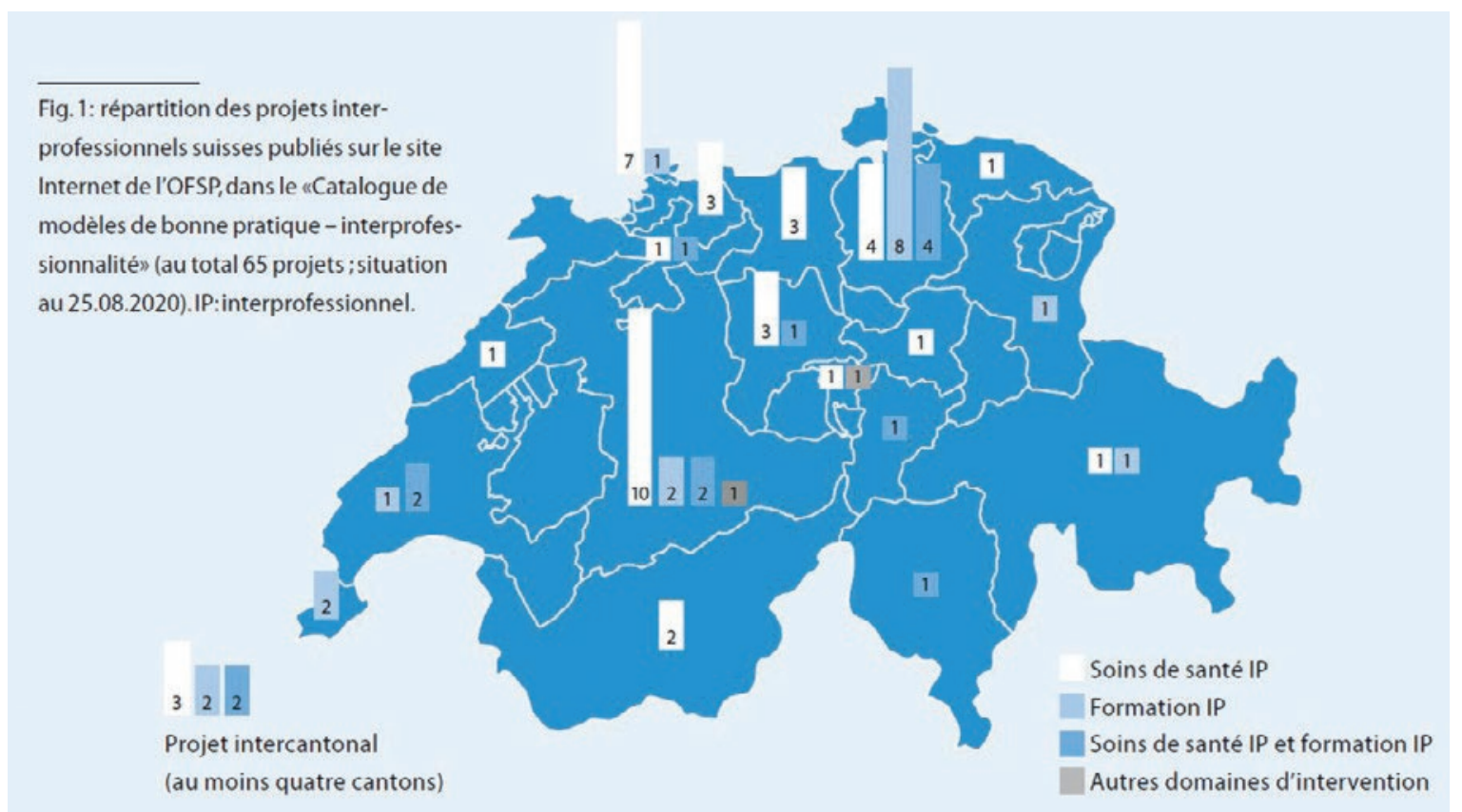

Figure 1: Cette carte indique la répartition cantonale des projets d'interprofessionnalité répertoriés par l'OFSP, en fonction de leurs axes prioritaires, état août 2021 [1] (৫ careum).

leux à tous ces défis majeurs. Elles peuvent néanmoins, en tant que quadruple aim [2], avoir d'importants effets positifs sur la santé de la population, les coûts de santé, l'expérience des patients et le bien-être du personnel de santé.

\section{Attention internationale}

Si la Suisse est encore à la traîne par rapport aux pays scandinaves dans le domaine de la formation et de la collaboration interprofessionnelles, de nombreuses évolutions positives ont contribué à améliorer nettement la situation, au point de susciter entre-temps une attention internationale.

\section{La Suisse est à la traîne par rapport aux pays scandinaves dans le domaine de la formation et de la collaboration interprofessionnelles.}

La Suisse compte de nombreux acteurs désireux de faire progresser la collaboration et la formation interprofessionnelles. Elle offre en outre des conditions-cadres visant à les soutenir. On peut par exemple citer l'Académie suisse des sciences médicales (ASSM), qui s'efforce de promouvoir l'interprofessionnalité de diverses manières, parmi lesquelles des prises de position, des manifestations, un prix de l'interprofessionnalité ou la Charte 2.0 «Collaboration interprofessionnelle dans le système de santé» [4].
L'Office fédéral de la santé publique (OFSP) a également mandaté 18 projets de recherche dans le domaine de la collaboration et de la formation interprofessionnelles pour un montant de trois millions de francs dans le cadre du programme de promotion "Interprofessionnalité dans le domaine de la santé» et en a résumé les résultats dans quatre Policy Briefs à la fin de la phase de promotion 2020 [5]. Ce programme a par ailleurs permis de créer un catalogue de modèles de bonnes pratiques, qui fait connaître les projets réussis dans toute la Suisse et incite à les reproduire [6]. Cette base de données contient actuellement 76 modèles interprofessionnels (fig. 1).

\section{Cadre légal}

La stratégie «Santé2020» du Conseil fédéral avait également pour objectif de traiter la dimension législative. C'est ainsi que la Suisse s'est dotée d'une nouvelle Loi sur les professions de la santé (LPSan) et a révisé en parallèle la Loi sur les professions médicales (LPMéd). Toutes deux incluent désormais l'interprofessionnalité, en postulant l'apprentissage avec d'autres groupes professionnels et en exigeant que les professionnels coordonnent les mesures prévues avec les autres acteurs du système de soins. Par conséquent, les procédures d'accréditation des filières de formation en médecine humaine de même que des professions de la santé dans les hautes écoles spécialisées devront par exemple tenir compte à l'avenir des offres interprofessionnelles. 


\section{Engagement de différentes institutions}

L'engagement des institutions de formation est également très réjouissant, les hautes écoles spécialisées dans le domaine de la santé se distinguant tout particulièrement puisqu'elles proposent depuis longtemps déjà des cadres d'apprentissage et d'enseignement interprofessionnels. La Haute école spécialisée bernoise et l'Université des Sciences Appliquées de Zurich disposent par exemple chacune d'un service spécialisé dédié à l'enseignement interprofessionnel. Toutes les facultés de médecine suisses proposent désormais elles aussi un tel enseignement, au moins facultatif, et les plans d'études des écoles supérieures et des écoles professionnelles du domaine de la santé prévoient également l'acquisition de compétences interprofessionnelles.

La fondation Careum est par ailleurs traditionnellement très engagée dans la formation et la collaboration interprofessionnelles à plusieurs niveaux. Un récent Careum Working Paper sur la formation interprofessionnelle analyse en détail la situation actuelle dans notre pays et, en se fondant sur des entretiens internationaux avec des experts et sur des ateliers nationaux, en formule des recommandations d'action et des mesures pour l'avenir de la formation interprofessionnelle en Suisse [1]. La fondation est partenaire du projet Station de formation clinique interprofessionnelle à Zurich (ZIPAS, www.zipas.ch), où des étudiants de différentes professions de santé et de différents niveaux de formation prennent en charge, sous supervision, des patients au sein d'équipes interprofessionnelles à l'hôpital [7].

\section{La stratégie "Santé2020» du Conseil fédéral avait également pour objectif de traiter la dimension législative.}

Cette image globalement positive en Suisse est soulignée par l'engagement de la plateforme interprofessionnalité (www.interprofessionnalite.ch), qui se concentre notamment sur la collaboration interprofessionnelle dans les soins de santé ambulatoires primaires, et par les offres de SwissIPE (www.swissipe.ch/ fr), qui propose des cours de leadership sur l'interprofessionnalité. Les étudiants aussi se montrent impliqués et motivés. Les étudiants des disciplines de la santé se sont ainsi réunis et ont fondé en 2020 la Swiss Health Alliance for Interprofessional Education (SHAPED). Leur but est de contribuer à ancrer plus fortement la formation interprofessionnelle dans les métiers en lien avec la santé.

\section{Pistes pour l'avenir}

Si l'on résume les résultats du Careum Working Paper [1] et du Policy Brief de l'OFSP sur la formation interprofessionnelle [5], on peut notamment retenir les pistes suivantes pour ces prochaines années en Suisse:

Il est nécessaire de créer un réseau national d'institutions de recherche, de pratique et d'enseignement sur l'interprofessionnalité et d'y intégrer les réseaux interinstitutionnels actuels, y compris locaux, afin d'exploiter de manière concertée les synergies existantes.

Nous avons besoin d'une compréhension commune des compétences et des objectifs d'apprentissage interprofessionnels, dont la formation théorique et pratique des professions de santé tienne obligatoirement compte au niveau national.

Les hautes écoles spécialisées de santé proposent depuis longtemps des cadres d'apprentissage et d'enseignement interprofessionnels.

Il faut concevoir et mettre en application des cours communs pour le corps enseignant mais aussi pour les patientes et patients (Patient as Teacher) dans la formation interprofessionnelle.

Cette dernière doit mettre l'accent sur des formats réalistes et adaptés à la pratique afin de garantir une formation de haute qualité. La mise en place à l'échelle nationale de stations de formation clinique interprofessionnelle (telles que ZIPAS) et les discussions sur le transfert vers le secteur ambulatoire pourraient apporter de précieuses contributions dans ce cadre.

L'interprofessionnalité doit être élargie à d'autres professions et disciplines impliquées dans les secteurs de la santé et social, comme les sciences du sport, le diagnostic de laboratoire biomédical, le travail social, la psychologie, mais aussi à d'autres acteurs, comme les proches des patients, les bénévoles dans les établissements de soins, les assurances, les organisations de patients ou les communes.

L'ancrage sur la durée en Suisse des infirmières en pratique avancée (advanced practice nurses) ouvrira de nouveaux champs d'action interprofessionnels.

\section{Tendances internationales}

Un coup d'œil au-delà de nos frontières et à la littérature internationale de ces dernières années montre en premier lieu que les pays d'Amérique du Nord et de Scandinavie ont toujours une longueur d'avance dans la formation et la collaboration interprofessionnelles. Il est néanmoins réjouissant de constater que de plus en plus de réseaux régionaux et/ou nationaux se 
forment un peu partout pour ancrer la collaboration interprofessionnelle de manière la plus globale possible [8].

Il apparaît aussi que la collaboration interprofessionnelle prend de l'ampleur dans le cadre de maladies spécifiques (diabète, démence, affections psychiques, etc.). Ces progrès vont de pair avec les efforts internationaux déployés pour rendre d'une part les soins de santé ambulatoires primaires plus interprofessionnels et d'autre part pour impliquer d'autres groupes professionnels ou parties prenantes afin de rompre davantage la compartimentation professionnelle des soins. Autre point intéressant: au niveau international, on ne s'intéresse plus seulement à la formation prégraduée,

\section{Des étudiants de différentes disciplines de la santé se sont réunis pour fonder la Swiss Health Alliance for Interprofessional Education.}

mais aussi postgraduée et continue afin d'intégrer plus directement l'interprofessionnalité dans la pratique des soins. A cette fin, des offres de formation interprofessionnelle en ligne sont, pandémie oblige, également envisagées [9].

\section{Ancrer l'interprofessionnalité}

Dans l'ensemble, la Suisse a réussi, avec l'aide de nombreux acteurs engagés, à donner à l'interprofessionnalité un positionnement prometteur au niveau politique, stratégique, législatif et au niveau des établissements de formation. Les parallèles avec les activités internationales confirment les efforts déployés dans notre pays. Ces prochaines années, il importera

\section{Série sur l'interprofessionnalité}

La collaboration entre les spécialistes de différentes professions de la santé est considérée comme un levier important afin de relever les défis du système de santé. Où en est la Suisse dans ce domaine? Quels avantages apporte l'interprofessionnalité et quelles en sont les limites? Nous éclairons le sujet sous divers angles dans une série d'articles. surtout de mettre à profit ce terreau favorable pour ancrer solidement et durablement l'interprofessionnalité dans la formation théorique et pratique suisses et pour assurer son financement à long terme.

Il sera en outre crucial que cet engouement se propage également de la formation prégraduée à la formation postgraduée et continue et à la pratique des soins, où les apprenants d'aujourd'hui, qui sont la main-d'œuvre de demain, devraient apprendre par l'exemple que la collaboration interprofessionnelle est une réalité vécue.

\section{Crédits photo}

Sulit Photos | Dreamstime.com

\section{Références}

1 Ulrich G, Amstad H, Glardon O, Kaap-Fröhlich S. La formation interprofessionnelle au sein du système de santé suisse: analyse de la situation, perspectives et roadmap; 2020 [18.10.2021]. https:// backend.careum.ch/sites/default/files/media/file/working-paper-9-formation-interprofessionnelle-suisse.pdf

2 Bodenheimer T, Sinsky C. From triple to quadruple aim: care of the patient requires care of the provider. Ann Fam Med. 2014;12(6): 573-6.

3 World Health Organization. Framework for action on interprofessional education and collaborative practice; 2010 [18.10.2021]. http://apps.who.int/iris/bitstream/10665/70185/1/WHO_HRH_ HPN_10.3_eng.pdf

4 SAMW. Charte 2.0 La collaboration interprofessionnelle dans le système de santé; 2020 [18.10.2021]. https://www.grea.ch/sites/ default/files/charte_interprofessionnalite_assm_2020.pdf

5 OFSP. Policy Briefs - Enseignements et recommandations pour la mise en œuvre spécifique aux groupes cibles; 2020 [18.10.2021]. https://www.bag.admin.ch/bag/fr/home/strategie-und-politik/ nationale-gesundheitspolitik/foerderprogramme-der-fachkraefteinitiative-plus/foerderprogramme-interprofessionalitaet/ policy-briefs-interprof.html

6 OFSP. Catalogue de modèles de bonne pratique - interprofession nalité: Office fédéral de la santé publique OFSP; 2020 [18.10.2021]. https://www.bag.admin.ch/bag/fr/home/strategie-und-politik/ nationale-gesundheitspolitik/foerderprogramme-der-fachkraefteinitiative-plus/foerderprogramme-interprofessionalitaet/ projektverzeichnis-modelle-guter-praxis.html

7 Bärlocher A, Caduff U, Staudacher D, Ulrich G. Lernen ohne Professionsgrenzen. Physioactive. 2020(6):19-24.

8 Khalili A, Thistlethwaite J, El-Awaisi A, Pfeifle A, Gilbert J, Lising D, et al. Guidance on Global Interprofessional Education and Collaborative Practice Research: Discussion Paper. A joint publication by InterprofessionalResearch.Global \& Interprofessional.Global. 2019 [18.10.2021]. https://interprofessionalresearch. global/global-ipecp-guidance/

9 Kaap-Fröhlich S, Ulrich G, Wershofen B, Ahles J, Behrend R, Handgraaf $M$, et al. Prise de position du comité de GMA - «Interprofessionelle Ausbildung in den Gesundheitsberufen» - Aktueller Stand und Zukunftsperspektiven. GMS J Med Educ (under Review). 2021. 